\section{ORIGINAL RESEARCH}

K.C. Young

B.S. Jahromi

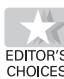

\title{
Does Current Practice in the United States of Carotid Artery Stent Placement Benefit Asymptomatic Octogenarians?
}

BACKGROUND AND PURPOSE: CAS or CEA for asymptomatic carotid stenosis is the focus of recently completed and ongoing randomized clinical trials. These techniques are widely utilized outside the setting of such trials. Therefore, our goal was to analyze the in-hospital stroke or death rates after CAS or CEA for asymptomatic stenosis that reflect current nationwide practice.

MATERIALS AND METHODS: Using sample-weighted ANOVA, we analyzed records from the 2006 and 2007 NIS, which are nationally representative cohorts for asymptomatic CAS or CEA. The primary outcome measure was a composite end point of in-hospital stroke, cardiac complications, or death. In-hospital stroke or death was a secondary outcome measure.

RESULTS: For $\geq 80$ years of age, the in-hospital stroke, cardiac complications, or death rate after CAS was $4.9 \%$, while the complication rate after CEA was $3.8 \%$. The stroke or death rate after CAS was $2.7 \%$ for $\geq 80$ years of age and was $1.5 \%$ after CEA for the same age group. Multivariate analysis showed that age $(\mathrm{OR}, 1.12 ; 95 \% \mathrm{Cl}, 0.97-1.3 ; P<.07)$ or procedure $(\mathrm{OR}, 1.12 ; 95 \% \mathrm{Cl}$, $0.99-1.27 ; P<.14)$ was not associated with the composite end point of in-hospital stroke, cardiac complications, or death. In contrast, CAS (OR, 1.28; $95 \% \mathrm{Cl}, 1.03-1.58)$ and female sex (OR, 1.23; $95 \% \mathrm{Cl}, 1.04-1.45)$ were independently associated with in-hospital stroke or death following asymptomatic carotid revascularization. Hospital charges and hospital costs were lower for CEA than CAS (2007 costs: $\$ 7779$ versus $\$ 12,104)$.

CoNCLUSIONS: CAS is independently associated with increased in-hospital stroke or death (excluding cardiac complications from the composite outcome). In those $\geq 80$ years of age, CAS as currently performed may not improve the natural history of asymptomatic carotid stenosis, because in-hospital stroke or death rates following CAS approached $3 \%$ in this group.

ABBREVIATIONS: ACAS = Asymptomatic Carotid Atherosclerosis Study; ACST = Asymptomatic Carotid Surgery Trial; ANOVA = analysis of variance; CAS = carotid artery stenting; $\mathrm{CC}=$ cardiac complications; $\mathrm{CEA}=$ carotid endarterectomy; $\mathrm{CHF}=$ congestive heart failure; $\mathrm{Cl}=$ confidence interval; COPD = chronic obstructive pulmonary disease; CREST = Carotid Revascularization Endarterectomy vs. Stenting Trial; ICD-9 = International Classification of Disease, Version 9; $\mathrm{MI}=$ myocardial infarction; NIS = Nationwide Inpatient Sample; NS = not significant after multivariate analysis; OR = odds ratio; OXVASC = Oxford Vascular Study; SE = standard error; SEM = standard error of the mean

$\mathbf{F}$ or asymptomatic subjects to benefit from carotid revascularization, 30-day stroke or death rates following CEA should be below 3\%. Similar arguments hold for CAS. ${ }^{1}$ Age increases morbidity and mortality after CAS, independent of symptom status. ${ }^{2}$ With many US practices increasingly adopting CAS, we wondered whether the morbidity and mortality rates of CAS as currently practiced were within published guidelines for carotid revascularization and were comparable with those of CEA. The NIS is a stratified dataset of discharges from nonfederal US hospitals, reflecting 2006 and 2007 practice patterns. $^{3}$

\section{Materials and Methods}

We used ICD-9 procedure codes to identify records for CAS (00.63) or CEA (38.12). ICD-9 diagnostic codes were used to iden-

\section{Received April 17, 2010; accepted after revision June 28}

From the Departments of Neurology (K.C.Y.), Surgery (K.C.Y.), Neurosurgery (B.S.J.), and Imaging Sciences (B.S.J.), University of Rochester Medical Center, Rochester, New York.

Please address correspondence to Kate C. Young, PhD, MPH, URMC Stroke Center, 601 Elmwood Ave, Box 681, Rochester, NY 14642; e-mail: kate_young@urmc.rochester.edu

Indicates open access to non-subscribers at www.ajnr.org

DOI 10.3174/ajnr.A2253 tify asymptomatic precerebral stenosis as an indication for CAS/ CEA (including 433.10 and 433.30, while excluding transient ischemic attack, 435.9 or 362.34 ). NIS datasets for 2006 and 2007 were analyzed. The main outcome was determined by the discharge code for death, ICD-9 diagnostic code for postoperative stroke (997.02), or ICD-9 diagnostic code for postoperative cardiac complications (997.1).

Comorbid conditions were identified by using clinical classification software and included renal disease, myocardial infarction, CHF, COPD, diabetes, hypertension, valve disease, and contralateral stenosis. ${ }^{4}$ Other characteristics included bilateral asymptomatic stenosis (ICD-9 diagnostic code 433.30), sex, race, tobacco use, atrial fibrillation (427.31), hyperlipidemia (272.x), hospital bed size, hospital teaching status, and hospital procedure volume by tertile. Data were analyzed by sample-weighted ANOVA by using SAS (SAS Institute, Cary, North Carolina). Variables with $P<.2$ after univariate analysis were included in the final multivariate model, except for year, which was included in the multivariate model.

Total charges per discharge record are also captured in the NIS. Hospital costs were calculated by using group average all-payer inpatient cost-to-charge ratios. 


\begin{tabular}{|c|c|c|c|c|c|}
\hline & \multirow[b]{2}{*}{ Overall } & \multicolumn{2}{|c|}{ Age } & \multicolumn{2}{|c|}{ Procedure } \\
\hline & & $<80$ & $80+$ & CAS & CEA \\
\hline \multicolumn{6}{|l|}{ Characteristics } \\
\hline Age (mean) (SEM) & $71.2(0.04)$ & & & $71.2(0.12)$ & $71.2(0.04$ \\
\hline$\%$ Total & & 79.7 & 20.3 & & \\
\hline$\%$ Age $80+$ & & & & 21.4 & 20.2 \\
\hline \multicolumn{6}{|l|}{ Sex } \\
\hline$\%$ Male & 57.5 & 58.3 & 54.6 & 60 & 57.2 \\
\hline \multicolumn{6}{|l|}{ Race $(\%)$} \\
\hline Nonwhite & 34.2 & 34.9 & 31.5 & 31.6 & 34.6 \\
\hline White & 65.8 & 65.1 & 68.5 & 68.4 & 65.4 \\
\hline \multicolumn{6}{|l|}{ Comorbid conditions (\%) } \\
\hline Contralateral stenosis & 17.2 & 17.2 & 17.4 & 19.7 & 16.8 \\
\hline Renal disease & 6.3 & 5.8 & 8.6 & 7.6 & 6.1 \\
\hline $\mathrm{Ml}$ & 50 & 49.7 & 51.2 & 56.9 & 49 \\
\hline CHF & 7.9 & 7.2 & 10.7 & 11.6 & 7.4 \\
\hline Atrial fibrillation & 8.8 & 7.1 & 15.2 & 9.0 & 8.7 \\
\hline Hyperlipidemia & 51.3 & 52.2 & 47.9 & 53.1 & 51.1 \\
\hline COPD & 18.4 & 19.2 & 15.5 & 17.7 & 18.6 \\
\hline Diabetes & 30.8 & 32.3 & 25 & 30.1 & 30.9 \\
\hline Hypertension & 78.6 & 78.5 & 79 & 74.9 & 79.2 \\
\hline Valve disease & 8.3 & 7.2 & 12.6 & 8.4 & 8.2 \\
\hline Tobacco use or history & 34.2 & 37.7 & 20.4 & 27.3 & 35.2 \\
\hline \multicolumn{6}{|l|}{ Hospital type (\%) } \\
\hline Nonteaching & 53.4 & 53 & 55.1 & 41.6 & 55.1 \\
\hline Teaching & 46.6 & 47 & 44.9 & 58.4 & 44.9 \\
\hline \multicolumn{6}{|l|}{ Hospital bed size (\%) } \\
\hline Small & 10.7 & 10.5 & 11.8 & 10.6 & 10.8 \\
\hline Medium & 22.8 & 22.8 & 22.9 & 19.2 & 23.4 \\
\hline Large & 66.4 & 66.7 & 65.3 & 70.2 & 65.9 \\
\hline
\end{tabular}

\begin{tabular}{|c|c|c|c|}
\hline & $\begin{array}{l}\text { CEA } \\
(\%)\end{array}$ & $\begin{array}{l}\text { CAS } \\
(\%)\end{array}$ & $\begin{array}{c}\text { Unadjusted OR } \\
(95 \% \mathrm{CI})\end{array}$ \\
\hline \multicolumn{4}{|l|}{ Primary end point } \\
\hline \\
\hline In-hospital stroke or death & 1.16 & 1.69 & $1.46(1.18-1.8)$ \\
\hline In-hospital stroke & 0.88 & 1.31 & $1.5(1.18-1.90)$ \\
\hline In-hospital CC & 1.86 & 2.15 & $1.16(0.96-1.39)$ \\
\hline In-hospital death & 0.39 & 0.57 & $1.46(1.02-2.09)$ \\
\hline
\end{tabular}

\section{Results}

CAS procedure volumes for asymptomatic stenosis were 17,750 for 2006 and 13,447 for 2007. CEA volumes were 108,276 and 110,119 for each year. Similar proportions of patients undergoing CAS $(21.4 \%)$ and CEA $(20.2 \%)$ were $\geq 80$ years of age. Cohort and hospital characteristics grouped by age or procedure are presented in Table 1. The likelihood of the primary end point of in-hospital stroke, cardiac complication, or death was not different between 2006 and 2007 (unadjusted OR, 0.99; 95\% CI, 0.89-1.1). Additionally, the likelihood of in-hospital stroke or death, as a secondary end point, was similar between 2006 and 2007 (unadjusted OR, 0.96; 95\% CI, 0.82-1.12; and OR adjusted for the procedure, $0.97 ; 95 \%$ CI, 0.83-1.14). Therefore, data from both years were combined for final analysis, with year as a covariate. The overall probability of in-hospital stroke, cardiac complications, or death was $2.9 \%$ after CEA and 3.66\% after CAS (Table 2; unadjusted OR, 1.27; 95\% CI, 1.1-1.47). The likelihood of inhospital stroke or death following CEA was $1.16 \%$, whereas
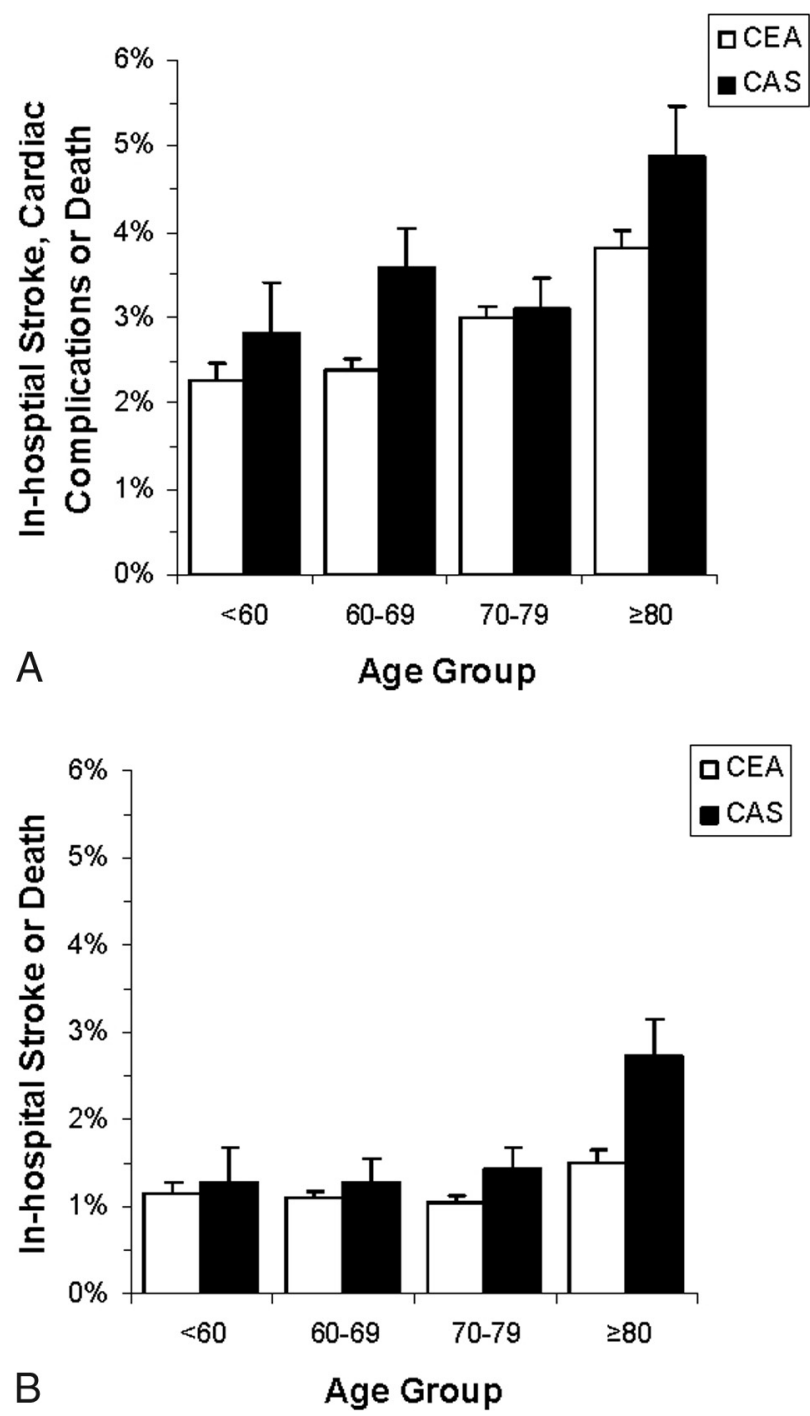

Fig 1. In-hospital complication rates following revascularization for asymptomatic stenosis by age and procedure. The likelihood of in-hospital stroke, cardiac complications, or death $(A)$ and the likelihood of in-hospital stroke or death (B) following CEA (white) or CAS (black) for 2006-2007 vary with age. Data are graphed as percentages with an adverse event plus SE.

the probability of a complication following CAS was $1.69 \%$ (unadjusted OR, 1.46; 95\% CI, 1.18-1.8).

We examined complication rates in patients $\geq 80$ years of age versus younger patients. On univariate analysis, the likelihood of in-hospital stroke, cardiac complications, or death after CEA was $2.7 \%$ for $\leq 79$ years of age and $3.8 \%$ for $\geq 80$ years of age (Fig $1 A$; unadjusted OR for age, 1.44; 95\% CI, 1.27-1.64). For each age category, the likelihood of in-hospital stroke, cardiac complications, or death was higher after CAS, with a probability of $3.3 \%$ for $\leq 79$ years of age and $4.9 \%$ for $\geq 80$ years of age (unadjusted OR for age, $1.5 ; 95 \%$ CI, 1.12 1.2). In-hospital stroke or death after CEA was $1.1 \%$ for $\leq 79$ years of age and $1.5 \%$ for $\geq 80$ years of age (Fig $1 B$; unadjusted OR, 1.41; 95\% CI, 1.15-1.72). In comparison, the in-hospital stroke or death rate after CAS was $1.4 \%$ for $\leq 79$ years of age and $2.7 \%$ for $\geq 80$ years of age (unadjusted OR, $1.96 ; 95 \% \mathrm{CI}$, 1.31-2.93), also exceeding that of CEA for each age group.

In multivariate analysis, use of CAS versus CEA for carotid 


\begin{tabular}{|c|c|c|c|c|}
\hline Characteristics & $\begin{array}{l}\text { OR for Stroke, CC, } \\
\text { or Death }(95 \% \text { CI) }\end{array}$ & $P$ Value & $\begin{array}{l}\text { OR for Stroke or } \\
\text { Death }(95 \% \mathrm{CI})^{\text {a }}\end{array}$ & $P$ Value \\
\hline Age $(\geq 80$ vs $<80)$ & $1.12(0.97-1.30)$ & .06 & $1.17(0.98-1.58)$ & .08 \\
\hline Procedure (CAS vs CEA) & $1.12(0.99-1.27)$ & .14 & $1.28(1.03-1.58)$ & .03 \\
\hline Sex (female vs male) & $0.97(0.87-1.09)$ & NS & $1.23(1.04-1.45)$ & .01 \\
\hline Year (2007 vs 2006) & $0.98(0.88-1.09)$ & NS & $0.95(0.81-1.12)$ & NS \\
\hline Race (white vs other) & Not included ${ }^{\mathrm{b}}$ & & $1.14(0.96-1.36)$ & NS \\
\hline \multicolumn{5}{|l|}{ Comorbidities } \\
\hline Renal disease & $1.79(1.52-2.11)$ & $<.0001$ & $2.67(2.15-3.36)$ & $<.0001$ \\
\hline $\mathrm{CHF}$ & $1.67(1.43-1.95)$ & $<.0001$ & $1.8(1.43-2.27)$ & $<.0001$ \\
\hline COPD & $1.25(1.09-1.42)$ & $<.01$ & $1.26(1.02-1.55)$ & .03 \\
\hline Diabetes & $0.84(0.74-0.94)$ & $<.01$ & $0.81(0.67-0.97)$ & .02 \\
\hline Valve disease & $1.40(1.20-1.63)$ & $<.0001$ & $1.23(0.96-1.57)$ & .1 \\
\hline Contralateral stenosis & $1.28(1.13-1.46)$ & $<.001$ & $1.3(1.06-1.58)$ & .01 \\
\hline Hypertension & $0.76(0.68-0.86)$ & $<.0001$ & $0.68(0.57-0.81)$ & $<.0001$ \\
\hline Tobacco & $0.65(0.57-0.74)$ & $<.0001$ & $0.57(0.46-0.7)$ & $<.0001$ \\
\hline Ml & $1.25(1.12-1.39)$ & $<.001$ & $1.01(0.85-1.19)$ & NS \\
\hline Atrial fibrillation & $2.83(2.48-3.24)$ & $<.0001$ & $1.62(1.3-2.03)$ & $<.0001$ \\
\hline Hyperlipidemia & $0.93(0.84-1.04)$ & NS & $0.76(0.64-0.89)$ & $<.001$ \\
\hline \multicolumn{5}{|l|}{ Hospital teaching status } \\
\hline Teaching vs nonteaching & $1.19(1.07-1.33)$ & $<.01$ & $1.12(0.95-1.31)$ & NS \\
\hline Hospital bed size & & $<.05$ & Not included ${ }^{\mathrm{b}}$ & \\
\hline Large vs small & $1.24(1.04-1.48)$ & & & \\
\hline Medium vs small & $1.26(1.03-1.53)$ & & & \\
\hline
\end{tabular}

adjusted for comorbid conditions and demographics (see "Materials and Methods").

b Variables were not included in the multivariate analysis because the univariate $P$ value was $>.2$.

\begin{tabular}{lllllr}
\hline \multicolumn{2}{l}{ Table 4: Economic data } & & & \\
\hline & \multicolumn{2}{c}{ Average } & & \multicolumn{2}{c}{ Median } \\
\cline { 2 - 3 } \cline { 5 - 6 } & CAS & CEA & & CAS & CEA \\
\hline 2006 & & & & \\
$\quad$ Cost & $\$ 15,461$ & $\$ 10,427$ & & $\$ 12,378$ & $\$ 7314$ \\
$\quad$ Charge & $\$ 39,852$ & $\$ 27,489$ & & $\$ 31,430$ & $\$ 18,235$ \\
2007 & & & & $\$ 12,104$ & $\$ 7779$ \\
$\quad$ Cost & $\$ 14,847$ & $\$ 10,900$ & & $\$ 32,533$ & $\$ 20,613$ \\
$\quad$ Charge & $\$ 41,030$ & $\$ 30,521$ & &
\end{tabular}

${ }^{a}$ In US dollars.

revascularization did not reach statistical significance for the composite end point of stroke, cardiac complications, or death (Table 3). The interaction between procedure and age dichotomized at 80 years of age was not significant. However, the odds of stroke or death as a secondary end point (excluding cardiac complications) were increased after CAS. This increase was independent of age, comorbidities, demographic factors, and year (OR, 1.28; 95\% CI, 1.03-1.58). The odds of in-hospital stroke or death were also increased for women. Several comorbid conditions (in particular, CHF and renal disease) also increased the likelihood of in-hospital stroke, cardiac complications or death, and in-hospital stroke or death. A history of MI, hospital bed size, and hospital teaching status increased the risk of an adverse event when cardiac complications were included in the composite end point. Race and year of procedure did not increase the odds of an adverse event.

Average and median hospital charges and costs for each procedure by year showed that CAS was more expensive than CEA (Table 4). These values exclude the physician fees. Finally, we examined the proportions of CEA versus CAS procedures that were performed at hospitals providing both procedures. In individuals $<80$ years of age, $19.8 \%$ underwent CAS while $80.2 \%$ underwent CEA. The proportion of CAS procedures increased in subjects $\geq 80$ years of age to $25.5 \%$ versus $74.5 \%$ who underwent CEA.

\section{Discussion}

Although the incidence of stroke, cardiac complications, or death was higher following CAS than following CEA regardless of age category, it did not reach statistical significance. However, the likelihood of stroke or death was higher after CAS than CEA for asymptomatic stenosis. In particular, inhospital stroke or death (without considering cardiac complications) after CAS for individuals $\geq 80$ years of age approached the $3 \%$ benchmark beyond which there is no net benefit for asymptomatic revascularization. ${ }^{1}$ The 30 -day rates for CAS are likely higher than the $2.7 \%$ in-hospital rate, which would be consistent with a recent meta-analysis reporting a $3.3 \%(2.6 \%-4.1 \%) 30$-day stroke or death rate following asymptomatic CAS. ${ }^{2}$ Age $>75-80$ years had a pooled relative risk of 1.93 (95\% CI, $1.66-2.24){ }^{2}$

For the asymptomatic subjects enrolled in CREST, the periprocedural composite end point of stroke, MI, or death was $3.5 \%$ (SE, $0.8 \%$ ) following CAS and 3.6\% (SE, $0.8 \%$ ) following CEA. ${ }^{5}$ These rates were not statistically significant. Our in-hospital composite end point of stroke, cardiac complications, or death after CEA was lower $(2.9 \%)$ than that in CREST, while the composite end point was similar following CAS. While death rates after each procedure from CREST and this study were comparable, our probability of in-hospital stroke following either CAS or CEA was lower than the periprocedural data of CREST. Cardiac complications, not MI specifically, were available from the NIS; thus, we cannot speculate further on this end point. Finally, our data were consistent with the CREST results showing that the probability of perioperative stroke or death was higher in the CAS arm than the CEA arm. 
During the timeframe of this study, the American Academy of Neurology guidelines did not recommend carotid revascularization for asymptomatic patients $\geq 75$ years of age, and the American College of Cardiology Foundation guidelines noted that there was no evidence to support CAS in asymptomatic patients of any age. ${ }^{6,7}$ In contrast to these guidelines, we found that asymptomatic octogenarians were nearly twice as likely to undergo CAS rather than CEA when compared with younger asymptomatic patients undergoing carotid revascularization in $2006-2007$ ( $11.4 \%$ versus $6.5 \%)$.

For 2006-2007, the in-hospital economics of carotid revascularization favored CEA, as does the long-term lifetime costeffectiveness. ${ }^{8}$ Physician fees were not included as part of the in-hospital costs. NIS charges represent what the hospital billed. Cost-to-charge ratios were used to estimate costs that include operating costs and capital-related costs. ${ }^{9}$

Two large trials of CEA for asymptomatic carotid stenosis found no benefit for patients $>68$ (ACAS) and 75 years of age (ACST). ${ }^{10}$ This is likely because subjects with asymptomatic stenosis derive modest benefits and only after several years. ${ }^{11}$ Recent studies, including OXVASC, imply an even lower stroke risk for optimal medically managed asymptomatic carotid stenosis than reported by ACAS or ACST. ${ }^{12}$ Our data, therefore, raise important questions regarding the current US practice of CAS in asymptomatic patients when considering the following: 1) A significant proportion are octogenarians, in whom CAS is not proved to be of benefit. 2) Paradoxically, asymptomatic octogenarians undergo CAS more frequently than younger patients. 3) The $2.7 \%$ in-hospital stroke or death rate of CAS in asymptomatic octogenarians suggests little or no improvement over the natural history of asymptomatic carotid disease, especially because this may actually underestimate the 30-day death and stroke rate, which should be lower than the benchmark 3\%. A further consideration may be made to recommend the best medical treatment in asymptomatic octogenarians, in lieu of any revascularization procedure.

Our study has several limitations. The NIS represents all discharges from a stratified sample of nonfederal US hospitals. The ICD-9 code represents cardiac complications rather than postoperative MI. Practice patterns, selection biases, and outcomes may be different in hospitals excluded from NIS. There is the potential for unmeasured confounders and selection bias for CAS or CEA beyond the covariates already included in this model. Discharge coding practices and accuracy are a limitation in administrative datasets. Nonetheless, our results include the strength of a nationally representative sample.

\section{Conclusions}

These in-hospital data suggest that routine CAS in asymptomatic octogenarians should await further comparative epidemiologic and clinical trial data regarding CAS, CEA, and medical management of asymptomatic carotid stenosis.

\section{References}

1. Goldstein LB, Adams R, Alberts MJ, et al. Primary prevention of ischemic stroke: a guideline from the American Heart Association/American Stroke Association Stroke Council-cosponsored by the Atherosclerotic Peripheral Vascular Disease Interdisciplinary Working Group; Cardiovascular Nursing Council; Clinical Cardiology Council; Nutrition, Physical Activity, and Metabolism Council; and the Quality of Care and Outcomes Research Interdisciplinary Working Group: the American Academy of Neurology affirms the value of this guideline. Stroke 2006;37:1583-633. Epub 2006 May 4

2. Touze E, Trinquart L, Chatellier G, et al. Systematic review of the perioperative risks of stroke or death after carotid angioplasty and stenting. Stroke 2009;40: e683-93

3. Nationwide Inpatient Sample. Healthcare Utilization Project. 2006-2007. Agency for Healthcare Research and Quality, Rockville, Maryland. http:// hcup-us.ahrq.gov/nisoverview.jsp. Accessed March 18, 2010

4. Clinical classification software for ICD-9-CM. Healthcare Cost and Utilization Project. Agency for Healthcare Research and Quality, Rockville, Maryland. www.hcup-us.ahrq.gov/toolssoftware/ccs/ccs.jsp. Accessed November 17, 2009

5. Brott TG, Hobson RW, Howard G, et al. Stenting versus endarterectomy for treatment of carotid-artery stenosis. N Engl J Med 2010;363:11-23

6. Chaturvedi S, Bruno A, Feasby T, et al. Carotid endarterectomy: an evidencebased review-report of the Therapeutics and Technology Assessment Subcommittee of the American Academy of Neurology. Neurology 2005;65:794801

7. Bates ER, Babb JD, Casey DE Jr, et al. ACCF/SCAI/SVMB/SIR/ASITN 2007 clinical expert consensus document on carotid stenting: a report of the American College of Cardiology Foundation Task Force on Clinical Expert Consensus Documents (ACCF/SCAI/SVMB/SIR/ASITN Clinical Expert Consensus Document Committee on Carotid Stenting). J Am Coll Cardiol 2007;49:126-70

8. Young KC, Holloway RG, Burgin WS, et al. A cost-effectiveness analysis of carotid artery stenting compared to endarterectomy. J Stroke Cerebrovasc Dis In press

9. Cost-to-Charge Ratio Files. Healthcare Cost and Utilization Project. 2007. Agency for Healthcare Research and Quality, Rockville, MD. www.hcup-us. ahrq.gov/db/state/costtocharge.jsp. Accessed March 18, 2010

10. Chambers BR, Donnan GA. Carotid endarterectomy for asymptomatic carotid stenosis. Cochrane Database Syst Rev 2005;CD001923

11. Halliday A, Mansfield A, Marro J, et al. Prevention of disabling and fata strokes by successful carotid endarterectomy in patients without recent neurological symptoms: randomised controlled trial. Lancet 2004;363:1491-502

12. Marquardt L, Geraghty OC, Mehta Z, et al. Low risk of ipsilateral stroke in patients with asymptomatic carotid stenosis on best medical treatment: a prospective, population-based study. Stroke 2010;41:e11-17 